5. Conclusion. It is worthy of remark that by means of the property (III), alone, the field of functions to which $F[f]$ is applicable may be considerably extended. If (III) is valid, $F[f]$ may be extended to all functions $f$ in $R$ summable Lebesgue. And if $\Phi_{1}(x) \equiv-\infty, \quad \Phi_{2}(x) \equiv+\infty$, the functional may be extended to all functions summable Lebesgue.

In fact, under (III), the functional has the same fundamental property as integration with respect to sequences $f_{n}(x)$ with limit $f(x)$; if the absolute continuity of $\int f_{n}(x) d x$ is uniform in $n$ and $\lim f_{n}(x)=f(x)$, it follows that $\lim F\left[f_{n}\right]$ exists and is independent of the choice of the sequence $f_{n}$. Hence $F[f]$ may be defined as the limit of $F\left[f_{n}\right]$. And thus in successive steps, and by writing $F\left[f_{1}\right]=F\left[f_{2}\right]$, if $f_{1}$ and $f_{2}$ differ only on a set of zero measure, the extension is completed.

The Rice Institute

\title{
THE EVALUATION OF CERTAIN DEFINITE INTEGRALS BY THE USE OF PROBABILITY FUNCTIONS*
}

\author{
BY W. D. BATEN
}

1. Introduction. The object of this paper is to present three methods of evaluating certain definite integrals by using probability functions. The first method consists in finding the probability law or function for the sum of $n$ independent variables, which are each subject to given probability laws, by Mayr's method $\dagger$ and comparing this with the probability for the same sum obtained by Dodd's method. On equating the two results thus obtained one often finds the value of certain integrals.

Method II consists in finding the probability law for $n$ independent variables, which is expressed as an integral, and then allowing $n$ to be equal to 1 .

\footnotetext{
* Presented to the Society, August 29, 1929.

$\dagger$ Karl Mayr, Wahrscheinlichkeitsfunktionen und ihre Anwendungen, Monatshefte für Mathematik und Physik, vol. 30.

$\ddagger \mathrm{E}$. L. Dodd, The frequency law of a function of one variable, this Bulletin, vol. 31 (1925), pp. 27-31; The frequency law of a function of variables with given frequency laws, Annals of Mathematics, (2), vol. 27, pp. 12-20.
} 
Method III is founded on setting up a probability function containing the integral to be evaluated as a factor, determining a second probability function defined over the same interval, and then finding a third probability function for a chosen relation of the variables under consideration. The integral need not be a factor of the first function, but should arise somewhere in the process.

The first two methods, as far as the writer knows, seem to be new.

2. Method I. Consider the integral

$$
\int_{0}^{\infty} \frac{\cos (u t-n k) d t}{\left(q^{2}+t^{2}\right)^{n r / 2}},
$$

where $k=r \tan ^{-1}(t / q)$, and $r>-1$. Let $x_{i}$ be taken arbitrarily out of the interval $(0, \infty)$ and let

$$
\frac{q^{r}}{\Gamma(r)} x_{i}^{r-1} e^{-q x_{i}} d x_{i}
$$

be the probability that $x_{i}$ lies in the interval $\left(x_{i}, x_{i}+d x_{i}\right)$. The probability function for the sum $x_{1}+x_{2}+x_{3}+\cdots+x_{n}=u$ can be written in two different forms following Mayr's or Dodd's method. These will be denoted hereafter by $P_{n}(u)$ and $F_{n}(u)$, respectively. We may then write, omitting $d u$,

$$
\begin{aligned}
& P_{n}(u)=\frac{q^{n r} u^{n r-1} e^{-q u}}{(n r-1) !} \\
& F_{n}(u)=\frac{q^{n r}}{\pi} \int_{0}^{\infty} \frac{\cos (u t-n k) d t}{\left(q^{2}+t^{2}\right)^{n r / 2}} .
\end{aligned}
$$

Equating these, we find

$$
\int_{0}^{\infty} \frac{\cos (u t-n k) d t}{\left(q^{2}+t^{2}\right)^{n r / 2}}=\frac{\pi u^{n r-1} e^{-q u}}{(n r-1) !} .
$$

Evaluating the integral, we obtain

$$
\int_{-\infty}^{\infty} e^{-h^{2} x(x-u)} d x .
$$

Let $x_{i}$ be taken arbitrarily out of the interval $(-\infty, \infty)$ and let the probability function for $x_{i}$ be 


$$
\frac{h}{\sqrt{ } \pi} e^{-h^{2} x_{i}^{2}} d x_{i} .
$$

The probability function for the sum $x_{1}+x_{2}=u$ is

$$
\begin{array}{ll}
P_{2}(u)=\frac{h^{2}}{\pi} \int_{-\infty}^{\infty} e^{-h^{2} u^{2}} e^{-2 h^{2} x(x-u)} d x, & \text { (Mayr's method) }, \\
F_{2}(u)=\frac{h}{(2 \pi)^{1 / 2}} e^{-h^{2} u^{2} / 2}, & \text { (Dodd's method) } .
\end{array}
$$

Hence we may write

$$
\int_{-\infty}^{\infty} e^{-2 h^{2} x(x-u)} d x=\frac{\sqrt{ } \pi}{h \sqrt{ } 2} e^{h^{2} u^{2} / 2} .
$$

Let $x_{i}$ be taken arbitrarily out of the interval $(-\infty, \infty)$ and let the probability function for $x_{i}$ be

$$
\frac{2 h}{\pi} \cdot \frac{1}{e^{h x_{i}}+e^{-h x_{i}}} .
$$

The probability function for the sum $x_{1}+x_{2}=u$ is

hence

$$
\begin{aligned}
& P_{2}(u)=\frac{4 h^{2}}{\pi^{2}} \int_{-\infty}^{\infty} \frac{e^{h u} e^{h x} d x}{\left(e^{2 h u}+e^{2 h x}\right)\left(e^{2 h x}+1\right)} \\
& F_{2}(u)=\frac{4 h^{2}}{\pi} \int_{0}^{\infty} \frac{\cos u t d t}{\left(e^{\pi t /(2 h)}+e^{-\pi t /(2 h)}\right)^{2}}=\frac{4 h^{2} u}{\pi^{2}\left(e^{h u}-e^{-h u}\right)}
\end{aligned}
$$

$$
\int_{-\infty}^{\infty} \frac{e^{h x} d x}{\left(e^{2 h u}+e^{2 h x}\right)\left(e^{2 h x}+1\right)}=\frac{u}{e^{2 h u}-1} .
$$

Let the probability function for $x_{i}$ be $1 / k$ for $-k / 2 \leqq x_{i} \leqq k / 2$, and zero elsewhere. The probability function for the sum $x_{1}+x_{2}=u$, by Mayr's method, is

$$
P_{2}(u)=\left\{\begin{array}{ccc}
0, & \text { for }(-\infty<u \leqq-k), \\
\frac{u+k}{k^{2}}, & \text { for } & (-k \leqq u \leqq 0), \\
\frac{-u+k}{k^{2}}, & \text { for } & (0 \leqq u \leqq k), \\
0, & \text { for } & (k \leqq u<\infty) ;
\end{array}\right.
$$


and by Dodd's method it is

$$
F_{2}(u)=\frac{4}{k^{2} \pi} \int_{0}^{\infty} \frac{\sin ^{2}(k t / 2) \cos (u t) d t}{t^{2}} .
$$

Hence we have

$$
\begin{aligned}
& \frac{4}{k^{2} \pi} \int_{0}^{\infty} \frac{\sin ^{2}(k t / 2) \cos (u t) d t}{t^{2}} \\
& =\left\{\begin{array}{ccc}
0, & \text { for } & (-\infty<u \leqq-k), \\
\frac{u+k}{k^{2}}, & \text { for } & (-k \leqq u \leqq 0), \\
\frac{-u+k}{k^{2}}, & \text { for } & (0 \leqq u \leqq k), \\
0, & \text { for } & (k \leqq u<\infty) .
\end{array}\right.
\end{aligned}
$$

In a similar manner, by using the probability law stated above for $x_{i}$, and the probability law for the sum $x_{1}+x_{2}+x_{3}$, or $x_{1}+x_{2}+x_{3}+x_{4}$, we get

$$
=\left\{\begin{array}{lc}
0, & \text { for } \quad\left(-\infty<u \leqq-\frac{3 k}{k^{3} \pi}\right), \\
\frac{1}{2 k^{3}}\left(u+\frac{3 k}{2}\right)^{2}, & \text { for } \quad\left(-\frac{3 k}{2} \leqq u \leqq-\frac{k}{2}\right), \\
\frac{1}{4 k^{3}}\left(-4 u^{2}+3 k^{2}\right), & \text { for } \quad\left(-\frac{k}{2} \leqq u \leqq \frac{k}{2}\right), \\
\frac{1}{2 k^{3}}\left(-u+\frac{3 k}{2}\right)^{2}, & \text { for } \quad\left(\frac{k}{2} \leqq u \leqq \frac{3 k}{2}\right), \\
0, & \text { for } \quad\left(\frac{3 k}{2} \leqq u<\infty\right) \\
\frac{2^{4}}{k^{4} \pi} \int_{0}^{\infty} \frac{\sin ^{4}(k t / 2) \cos (u t) d t}{t^{4}}
\end{array}\right.
$$




$$
\begin{cases}0, & \text { for }(-\infty<u \leqq-2 k), \\ \frac{1}{6 k^{4}}(u+2 k)^{3}, & \text { for }(-2 k \leqq u \leqq-k), \\ \frac{1}{6 k^{4}}\left(-3 u^{3}-6 k u^{2}+4 k^{3}\right), & \text { for } \quad(-k \leqq u \leqq 0), \\ \frac{1}{6 k^{4}}\left(3 u^{3}-6 k u^{2}+4 k^{3}\right), & \text { for } \quad(0 \leqq u \leqq k), \\ \frac{1}{6 k^{4}}(-u+2 k)^{3}, & \text { for } \quad(k \leqq u \leqq 2 k), \\ 0, & \text { for } \quad(2 k \leqq u<\infty) .\end{cases}
$$

By continuing this process, a probability function for the sum of $n$ independent variables is obtained, which is continuous and has different analytic expressions in different intervals of its existence, yet which can be expressed as a single definite integral.

3. Method II. If $f_{i}\left(x_{i}\right)=3\left(1-x_{i}{ }^{2}\right) / 4$ is the probability law for $x_{i}$ for $-1 \leqq x_{i} \leqq 1$, and zero elsewhere, then the probability law for the sum $x_{1}+x_{2}+x_{3}+\cdots+x_{n}=u$, is

$$
F_{n}(u)=\frac{3^{n}}{\pi} \int_{0}^{\infty} \frac{(\sin t-t \cos t) \cos (u t) d t}{t^{3 n}} .
$$

For $n=1$, this integral is evidently $f_{i}(u)$. Hence

(6) $\int_{0}^{\infty} \frac{(\sin t-t \cos t) \cos (u t) d t}{t^{3}}=\frac{\pi}{4}\left(1-u^{2}\right),(-1 \leqq u \leqq 1)$.

In particular, for $u=0,1$, we have

$$
\begin{aligned}
& \int_{0}^{\infty} \frac{(\sin t-t \cos t) d t}{t^{3}}=\frac{\pi}{4} \\
& \int_{0}^{\infty} \frac{(\sin t-t \cos t) \cos t d t}{t^{3}}=0 .
\end{aligned}
$$

If the probability function for $x_{i}$ is $1 /(2 k),-k \leqq x_{i} \leqq k$, and zero elsewhere, where $k$ is a certain constant, then the law for the sum of $n$ independent variables is, according to Dodd's method, 


$$
F_{n}(u)=\frac{1}{\pi} \int_{0}^{\infty} \frac{(\sin k t)^{n} \cos (u t) d t}{k^{n} t^{n}} .
$$

If we set $n=1$ and $u=0$, we find the well known integrals

$$
\begin{aligned}
\int_{0}^{\infty} \frac{\sin (k t) \cos (u t) d t}{t}=\frac{\pi}{2}, \quad(-k \leqq u \leqq k), \\
\int_{0}^{\infty} \frac{\sin (k t) d t}{t}=\frac{\pi}{2}, \quad(k>0) .
\end{aligned}
$$

Let the probability law for $x_{i}$ be $\left(\cos x_{i}\right) / 2$ for $-\pi / 2 \leqq x_{i} \leqq \pi / 2$, and zero elsewhere; the law for the sum of $n$ variables is

$$
F_{n}(u)=\frac{1}{\pi} \int_{0}^{\infty} \frac{\cos ^{n}(t \pi / 2) \cos (u t) d t}{\left(1-t^{2}\right)^{n}} .
$$

If we set $n=1$, this becomes

$$
\int_{0}^{\infty} \frac{\cos (t \pi / 2) \cos (u t) d t}{\left(1-t^{2}\right)}=\frac{\pi}{2} \cos u, \quad(-\pi / 2 \leqq u \leqq \pi / 2) .
$$

In particular, with $u=0$,

$$
\int_{0}^{\infty} \frac{\cos (t \pi / 2) d t}{\left(1-t^{2}\right)}=\frac{\pi}{2} .
$$

If the probability law for $x_{i}$ is

$$
\frac{h}{\sqrt{ } \pi} e^{-h^{2} x_{i}},
$$

for $\left(-\infty<x_{i}<\infty\right)$, then the law for the sum of the squares, $x_{1}^{2}+x_{2}^{2}+x_{3}^{2}+\cdots+x_{n}^{2}=u$, is

$$
F_{n}(u)=\frac{h^{n}}{\pi} \int_{0}^{\infty} \frac{\cos (u t-n m) d t}{\left(h^{4}+t^{2}\right)^{1 / 4}}, \quad \tan 2 m=\frac{t}{h^{2}} .
$$

For $n=1$, we find

$$
\frac{h}{\pi} \int_{0}^{\infty} \frac{\cos (u t-m) d t}{\left(h^{4}+t^{2}\right)^{1 / 4}}=\frac{h e^{-h^{2} u}}{(\pi u)^{1 / 2}} .
$$

When written in full, this becomes 


$$
\begin{gathered}
\frac{h}{2 \pi} \int_{0}^{\infty} \frac{\left[\left(h^{4}+t^{2}\right)^{1 / 2}+h^{2}\right]^{1 / 2} \cos (u t)-\left[\left(h^{4}+t^{2}\right)^{1 / 2}-h^{2}\right]^{1 / 2} \sin (u t) d t}{\left(h^{4}+t^{2}\right)^{1 / 4}} \\
=\frac{h e^{-h^{2} u}}{(\pi u)^{1 / 2}} .
\end{gathered}
$$

4. Method III. We shall now show how to evaluate the integral

$$
J=\int_{0}^{\infty} \frac{e^{-p x} \sin (q x) d x}{x}
$$

Let

$$
f(x)=\frac{1}{J} \cdot \frac{e^{-p x} \sin q x}{x}, \quad g(y)=e^{-y},
$$

respectively, be the probability functions for the variables $x$ and $y$ for $0 \leqq x, y<\infty$, and let $y=x s$; then the probability function for $s$ is*

$$
P(s)=\frac{1}{J} \int_{0}^{\infty} \frac{e^{-p x} \sin (q x) e^{-s x} x d x}{x}=\frac{1}{J} \cdot \frac{q}{q^{2}+(p+s)^{2}} .
$$

Since $P(s)$ is a probability function for the interval $(0, \infty)$, we have $\int_{0}^{\infty} P(s) d s=1$. Therefore

$$
\frac{1}{J} \int_{0}^{\infty} \frac{q d s}{q^{2}+(p+s)^{2}}=\frac{1}{J}\left(\frac{\pi}{2}-\tan ^{-1} \frac{p}{q}\right)=\frac{1}{J}\left(\tan ^{-1} \frac{q}{p}\right) .
$$

Hence

$$
J=\int_{0}^{\infty} \frac{e^{-p x} \sin (q x) d x}{x}=\tan ^{-1} \frac{q}{p} .
$$

Consider the integral

$$
\int_{0}^{\infty} \frac{d s}{1+s^{4}}
$$

Let

$$
f(x)=\frac{\sqrt{ } 2}{\pi} \cdot \frac{\sin x}{\sqrt{ } x}, \quad g(y)=\frac{2}{\sqrt{ } \pi} e^{-y^{2}},
$$

where $x$ and $y$ lie in the interval $(0, \infty)$, and let $y=s \sqrt{ } x$. Then we have

* See the second footnote on p. 433. 


$$
P(s)=\frac{2 \sqrt{ } 2}{\pi} \int_{0}^{\infty} \frac{e^{-x s^{2}} \sqrt{ } x \sin x d x}{\sqrt{ } x}=\frac{2 \sqrt{ } 2}{\pi\left(1+s^{4}\right)} .
$$

Since we know that $\int_{0}^{\infty} P(s) d s=1$, we may write

$$
\int_{0}^{\infty} \frac{d s}{1+s^{4}}=\frac{\pi}{2 \sqrt{ } 2} .
$$

We shall now prove that

$$
\int_{0}^{\infty} \frac{\cos x d x}{x+a}=\int_{0}^{\infty} \frac{x e^{-a x} d x}{1+x^{2}}
$$

Let

$$
f(x)=\frac{1}{J} \cdot \frac{\cos x}{x+a}, \quad g(y)=e^{-\dot{y}},
$$

for $0 \leqq x, y<\infty$, where $J$ is the integral on the left of the above equality. The probability law for $s$, where $y=(x+a) s$, is

(21) $P(s)=\frac{1}{J} \int_{0}^{\infty} \frac{\cos x e^{-(x+a) s}(x+a) d x}{(x+a)}=\frac{1}{J} \cdot \frac{s e^{-a s}}{1+s^{2}}$.

But $\int_{0}^{\infty} P(s) d s=1$. Hence

or

$$
\frac{1}{J} \int_{0}^{\infty} \frac{s e^{-a s} d s}{1+s^{2}}=1
$$

$$
\int_{0}^{\infty} \frac{s e^{-a s} d s}{1+s^{2}}=J=\int_{0}^{\infty} \frac{\cos s d s}{s+a}
$$

which agrees essentially with (20).

The University of Michigan 